\title{
Spin orientation and Spin-Hall effect induced by tunneling electrons
}

\author{
A.G. Mal'shukov ${ }^{1,2,3}$ and C.S.Chu ${ }^{2}$ \\ ${ }^{1}$ Institute of Spectroscopy, Russian Academy of Science, 142190 Troitsk, Moscow oblast, Russia \\ ${ }^{2}$ Department of Electrophysics, National Chiao Tung university, Hsinchu 30010, Taiwan \\ ${ }^{3}$ National Center for Theoretical Sciences, Physics Division, Hsinchu 30043, Taiwan
}

\begin{abstract}
It is shown that a flux of unpolarized electrons across a symmetric double barrier quantum well induces a spin polarization inside the well. Besides, the transmitted current acquires a spin polarized component and the spin-Hall current flows in the planar direction. These phenomena are due to a combined effect of Dresselhaus interaction and the spin-orbit interaction induced by gradients of heterostructure material parameters. In contrast to previous studies of the spin filtering effect, we predict that it can be observed in case of an isotropic distribution of incident electrons.

PACS numbers: 71.70.Ej, 72.25.Dc, 73.40.Lq
\end{abstract}

\section{INTRODUCTION}

The spin-orbit interaction (SOI) is a fundamental quantum relativistic phenomenon which recently attracted much interest in connection with spin transport of electrons in semiconductors and metals. Due to SOI, an electric field can influence the spin degree of freedom, thereby giving rise to a number of transport phenomena which have potential for application in spintronics. One of them is the spin-Hall effect (SHE), which recently has been intensively studied both theoretically (for a review see 1) and experimentally,$\frac{2,3}{*}$ A standard system to study this effect is a 2D electron gas confined within a quantum well. Due to SHE the electric current in the quantum well gives rise to a perpendicular flux of the spin polarization, as well as to the out of plane spin density near sample edges. Closely related to SHE is the electric spin orientation, which is a bulk inplane spin polarization induced by the DC electric current $\underline{4}$ In both cases SOI is realized either via impurity scattering, or due to an intrinsic spin-orbit coupling mechanism. The latter consists of two parts. The first one is the Dresselhaus interaction ${ }^{\frac{5}{5}}$ which is inherent to all zinc-blende semiconductors. The second contribution is determined by gradients of material parameters and the electric potential

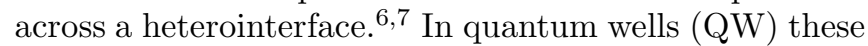
interactions are averaged with wave functions of confinement coordinate in the heterostructure growth direction. After such an averaging procedure the second term transforms into the Rashba $\mathrm{SOI}^{8}$ which is not zero only in asymmetric in $z$-direction (growth direction) heterostructures. The Rashba and averaged Dresselhaus interactions are basic SOI widely used in works on SHE, as well as in works on spin-dependent transport in general.

In this work we will consider spin-orbit effects on electron transport from a different point of view. Namely, we will consider the electric current parallel to the growth direction, rather than parallel to the quantum well. An appropriate model for studying such a situation is a double barrier quantum well. A principal difference from the conventional 2D system is that one can not use neither Rashba nor Dresselhaus interactions averaged over
QW confinement. For example, a part of the latter interaction, which is proportional to the $z$-component of the electron momentum operator, turns to 0 when averaged with a wave function of a confined state in the well. At the same time it is finite for tunneling states. Considering these states it is also easy to see that SOI associated with electric fields at heterointerfaces does not reduce to the single parameter Rashba interaction. The explicit dependence on $z$ of the initial spin-orbit interaction becomes important. To make this point more clear we deliberately considered a symmetric heterostructure, where Rashba SOI is zero. For such a model of a symmetric double barrier quantum well we found out that the electric current in the $z$-direction induces a parallel to the $z$-axis spin density, as well as a current of spins polarized in a planar direction. The spin current flows parallel to heterointerfaces. The former effect is an ana$\log$ of the electric spin orientation, while the latter is the spin-Hall effect. Besides, we found the transmittance of the double barrier structure to be dependent on the spin orientation. A similar spin filtering effect has been considered before within various models $\frac{9.10}{10}$ It was shown there that this effect can be observed only in case of an anisotropic distribution of incident electrons. It was suggested to create such a distribution applying a planar electric field. In contrast to these works, we predict the spin filtering effect for an isotropic distribution of electrons tunneling through a symmetric double barrier structure. Moreover, our spin filter makes electrons to be polarized in z-direction, instead of the planar polarization in Ref. 9, 10. Such fundamental distinctions arise from the $\sim k_{z}$ term of the Dresselhaus interaction which has been neglected in Ref. 10.

The general Hamiltonian of the problem will be derived in Sec.II. In Sec. III we will present our results related to the spin-Hall effect, spin orientation and the spin filtering effect. A brief conclusion is presented in Sec. IV.

\section{SPIN-ORBIT HAMILTONIAN}

Let us consider a quantum well $(\mathrm{QW})$ of the width $2 d$ separated from the left $(z<-d-b)$ and right $(z>d+b)$ 
parts of a doped semiconductor system by two equal barriers of the thickness $b$. These parts are assumed to be thermal reservoirs with respective chemical potentials $\mu_{l}$ and $\mu_{r}$. Besides a system homogeneous in $x, y$ directions, we will also consider an electron gas confined in the $y$ direction. For simplicity, the adiabatic case will be considered when the confinement width $w$ slowly increases from QW towards reservoirs. This situation is realized when the confinement is achieved by depleting the electron gas with the help of electrodes on top of QW.

The spin-orbit interaction in such a system is represented by two Hamiltonians $H_{s o}^{(1)}$ and $H_{s o}^{(2)}$. The former is the Dresselhaus SOI, while the latter is SOI due to change of the band gap width and other material parameters across heterointerfaces. Usually, in narrow gap semiconductors $H_{s o}^{(2)}$ is much stronger. Therefore, we will consider the Dresselhaus interaction within the first order perturbation theory. Only the part of $H_{s o}^{(1)}$ which is proportional to the $z$-component of the electron momentum operator will be taken into account. Hence, for [001] growth direction

$$
H_{s o}^{(1)}=-i \gamma \sigma_{z}\left(k_{x}^{2}-k_{y}^{2}\right) \frac{\partial}{\partial z},
$$

where $\sigma_{z}$ is the Pauli matrix and $\gamma$ is the coupling parameter, which is assumed to be $z$-independent. An important property of Hamiltonian (1) is that its expectation values taken with tunnelling states incident from the left and from the right reservoirs have opposite signs, while it does not change sign when $k_{x}, k_{y} \rightarrow-k_{x},-k_{y}$. Alternatively, other parts of the Dresselhaus SOI, which have been omitted in (11), are even functions of $\hat{k}_{z}$ and odd functions of $k_{x}, k_{y}$. Since, the interaction represented by $H_{s o}^{(2)}$ is of the same symmetry, the omitted terms of $H_{s o}^{(1)}$ do not add particularly new qualitative features to spin dependent electron tunneling, as well as to other effects considered below. At the same time, the symmetry difference of (1) and $H_{s o}^{(2)}$ has important consequences for these effects. This is also the main reason why the results of Ref. 10, where interaction (10) has been neglected, are qualitatively different from those presented below.

Following Ref. 6 the Hamiltonian $H_{s o}^{(2)}$ can be written as

$$
H_{s o}^{(2)}=\frac{1}{k_{\|}}\left(\sigma_{x} k_{y}-\sigma_{y} k_{x}\right) h(z)
$$

where

$$
h(z)=k_{\|} \frac{\partial \beta}{\partial z}
$$

and $k_{\|}=\sqrt{k_{x}^{2}+k_{y}^{2}}$. The parameter $h(z)$ denotes SOI strength which varies across the heterostructure depending on semiconductor material parameters and the electric potential. Ignoring the electron energy, which is much less than the gap value, $\beta(z)$ can be written as ${ }^{6}$

$$
\beta(z)=\frac{1}{2 m(z)} \frac{\Delta(z)}{3 E_{g}(z)+\Delta(z)},
$$

where $E_{g}(z)$ and $\Delta(z)$ are respective values of the band gaps and split off energies.

The major effect of SOI (11) is that it gives rise to spin precession around the $z$-axis. This precession takes place during particle transmission through the double barrier structure. Since the width of this structure is small in comparison with the spin precession length, the effect of the spin precession is expected to be small. In order to get explicitly the corresponding small parameter, the Hamiltonian can be transformed using an appropriate unitary transformation. Taking into account that the kinetic energy operator in $z$-direction is

$$
\frac{1}{2} \frac{\partial}{\partial z} \frac{1}{m(z)} \frac{\partial}{\partial z}
$$

one can apply the unitary transformation

$$
H \rightarrow U^{-1} H U
$$

with

$$
U=e^{i \sigma_{z} \vartheta(z)}
$$

where

$$
\vartheta(z)=-\gamma\left(k_{x}^{2}-k_{y}^{2}\right) \int_{0}^{z} m(z) d z .
$$

This transformation removes (1) from the Hamiltonian. At the same time, applying it to (2) one obtains, up to the linear in $\vartheta(z)$ terms, the spin orbit interaction

$$
H_{s o}=H_{s o}^{(2)}+\frac{2 \vartheta(z)}{k_{\|}}\left(\sigma_{x} k_{x}+\sigma_{y} k_{y}\right) h(z) .
$$

For the model under consideration, with rectangular symmetric barriers and a rectangular QW, $h(z)$ becomes

$$
\begin{aligned}
h(z) & =k_{\|}\left[\left(\beta_{r}-\beta_{b}\right)(\delta(z-b-d)-\delta(z+b+d))\right. \\
& \left.+\left(\beta_{b}-\beta_{w}\right)(\delta(z-d)-\delta(z+d))\right] .
\end{aligned}
$$

The parameters $\beta_{r}, \beta_{b}$ and $\beta_{w}$ denote SOI strengths for reservoirs, barriers and QW, respectively.

The transmission wave functions are represented by two sets of functions incident from the left $\left(\psi^{l}\right)$ and from the right $\left(\psi^{r}\right)$ of the double barrier structure. In the zeroth order, when the second term in (9) is ignored, these functions can be conveniently written using the chiral basis. In case of the quantum wire confinement this basis corresponds to the spin quantization axis directed along the $y$-axis. Outside the double barrier structure the scattering eigenstates are represented by incident, transmitted and reflected plane waves, with transmission and reflection amplitudes $t_{\sigma}^{l / r}$ and $r_{\sigma}^{l / r}$, respectively, where $\sigma=1,2$ denotes the spin projection in the corresponding chiral basis. This projection is conserved upon the scattering, as far as the second term in (9) is neglected. The wave vector of the scattering states is denoted as $k=\sqrt{2 m_{r}\left(E-E_{\|}\right)}$, where $m_{r}$ is the electron effective mass in the left and right reservoirs, $E$ is the total energy and $E_{\|}$is the energy of motion in $x, y$ directions. 


\section{SPIN CURRENT AND SPIN POLARIZATION}

\section{A. Spin current}

The non zero spin current $J_{n}^{s}$, where $s$ and $n$ denote the spin polarization and current direction, respectively, can be calculated already in the zeroth order with respect to $\vartheta(z)$, while the latter will be important below in calculation of the spin density and spin dependent transmission. Hence, in this subsection we entirely neglect the presumably weak Dresselhaus SOI. Let us take the chiral component of $J_{n}^{s}$. That means that we look for the flux of spins polarized perpendicular to the flux direction, that can be expressed as $J_{n}^{s}=J \varepsilon^{s n z}$, where $\varepsilon^{s n z}$ is the antisymmetric tensor. Using the conventional definition of the spin current operator $\hat{J}_{n}^{s}=\left\{v_{n}, \sigma_{s} / 4\right\}$, where the spin dependent part of

$$
v_{n}=\frac{k_{n}}{m(z)}+\varepsilon^{n i z} \sigma_{i} \frac{h(z)}{k_{\|}}
$$

is obtained from (2) and (3), the spin current density can be written as

$$
\begin{aligned}
J_{n}^{s}(z) & =\frac{\varepsilon^{s n z}}{2} \sum_{\mathbf{k}_{\|}, \gamma} \int_{0}^{\infty} \frac{d k}{2 \pi}\left[\frac{k_{\|}}{2 m^{*}(z)}\left(\left|\psi_{1}^{\gamma}\right|^{2}-\left|\psi_{2}^{\gamma}\right|^{2}\right)\right. \\
& \left.+\frac{h(z)}{k_{\|}}\left(\left|\psi_{1}^{\gamma}\right|^{2}+\left|\psi_{2}^{\gamma}\right|^{2}\right)\right] n_{F}^{\gamma}(E)
\end{aligned}
$$

where $\gamma=l, r$ and $n_{F}^{\gamma}(E)$ is the Fermi distribution function for the left and right reservoirs. The first term in square brackets represents the bulk spin current density distributed in QW, barriers and outside, while the second term is the "surface" term which, according to (10), is finite only on heterostructure interfaces. From Eq.(12) it becomes immediately evident that the spin current is not zero in the equilibrium state when $n_{F}^{l}(E)=n_{F}^{r}(E)$. For example, the surface current at each interface is given by $\rho(z) \Delta \beta(z)$, where $\rho(z)$ is the local equilibrium electron density and $\Delta \beta(z)$ is the difference of the spin-orbit coupling parameters $\beta$ on both sides of the interface, as follows from (10). In a symmetric QW the surface currents on opposite interfaces flow in opposite directions, so that they cancel each other. It is easy to see that the total equilibrium current, obtained by integration of Eq. (12) over $z$, is identically zero in case of a symmetric heterostructure. That follows from the symmetry relation $\psi_{1}^{l / r}(z)=\psi_{2}^{r / l}(-z)$ which makes $J_{n}^{s}(z)$ to be an odd function of $z$. At the same time, one can not expect the total current to be zero in an asymmetric heterostructure, as has been shown by Rashba ${ }^{11}$ for confined states. It should be noted that Rashba found that the total equilibrium current is cubic with respect to the spin-orbit coupling constant, while the current density given by Eq. (12) is linear. The latter becomes evident from the above expression for the surface current. That means that, at least, linear and quadratic terms vanish after integration of (12) over $z$.

By convention, the "nonequilibrium" current could be defined as a part of (12) which is proportional to $n_{F}^{l}-n_{F}^{r}$. In symmetric QW this current density is an even function of $z$ and, hence, the corresponding total nonequilibrium current is finite. However, one can not define unambiguously the dissipative part of the spin current using only its definition (12). Calculation of the spin accumulation at the sample boundary would be helpful to clarify the physical meaning of Eq. (12).

\section{B. Spin orientation}

In order to calculate the spin density induced by the tunneling current, the second term in (9) must be taken into account. It causes spin flip processes upon transmission and reflection of particles incident onto the double barrier structure. Therefore, we label spin variables of wave functions by two indices, as $\psi_{\alpha \beta}$, where $\alpha$ denotes the spin polarization of the incident wave. Treating such functions as matrices, the spin density can be expressed as

$$
\mathbf{S}(z)=\frac{1}{2} \sum_{\mathbf{k}_{\|}, \gamma} \int_{0}^{\infty} \frac{d k}{2 \pi} \operatorname{Tr}\left[\psi^{\gamma+} \boldsymbol{\sigma} \psi^{\gamma}\right] n_{F}^{\gamma}(E)
$$

where $\boldsymbol{\sigma}=\left(\sigma_{x}, \sigma_{y}, \sigma_{z}\right)$ is the vector of Pauli matrices. We calculate (13) in the first order perturbation theory with respect to $\vartheta(z)$. Since a commutator of two terms in (9) is proportional to $\sigma_{z}$, one should expect the $z$-component of $\mathbf{S}(z)$ to be finite. Further, the time inversion symmetry dictates

$$
\sum_{\gamma=l, r} \operatorname{Tr}\left[\psi_{\mathbf{k}_{\|}}^{\gamma+} \boldsymbol{\sigma} \psi_{\mathbf{k}_{\|}}^{\gamma}\right]=-\sum_{\gamma=l, r} \operatorname{Tr}\left[\psi_{-\mathbf{k}_{\|}}^{\gamma+} \boldsymbol{\sigma} \psi_{-\mathbf{k}_{\|}}^{\gamma}\right] .
$$

Applying this relation to Eq. (13) the latter is transformed to

$$
S_{z}(z)=\sum_{\mathbf{k}_{\|}} \int_{0}^{\infty} \frac{d k}{4 \pi} \operatorname{Tr}\left[\psi^{l+} \sigma_{z} \psi^{l}\right]\left(n_{F}^{l}(E)-n_{F}^{r}(E)\right)
$$

It immediately follows from this expression that the spin density is zero in the equilibrium state.

The first order correction to the wave function can be written in terms of the retarded Green function, so that

$$
\psi_{\alpha \beta}^{l}(z)=\psi_{\alpha}^{l}(z) \delta_{\alpha \beta}+\int d z^{\prime} G_{\beta}\left(z, z^{\prime}\right) V_{\beta \alpha}\left(z^{\prime}\right) \psi_{\alpha}^{l}\left(z^{\prime}\right),
$$

where $V_{\beta \alpha}\left(z^{\prime}\right)$ is a matrix element of the second term in (9) and $\psi_{\alpha}^{l}(z)$ is the unperturbed wave function. In its turn, the Green function is given by

$$
\begin{aligned}
G_{\beta}\left(z, z^{\prime}\right) & =-i \frac{m_{r}}{k t_{\beta}}\left[\psi_{\beta}^{l}(z) \psi_{\beta}^{r}\left(z^{\prime}\right) \theta\left(z-z^{\prime}\right)\right. \\
& \left.+\psi_{\beta}^{r}(z) \psi_{\beta}^{l}\left(z^{\prime}\right) \theta\left(z^{\prime}-z\right)\right] .
\end{aligned}
$$


In the considered case of a symmetric heterostructure, it is easy to see that the transmission coefficient $t_{1}=$ $t_{2} \equiv t$. At the same time, the reflection is spin dependent through its phase. We note that, according to (8), the matrix elements $V_{\beta \alpha}$ are proportional to $k_{x}^{2}-k_{y}^{2}$. After integration in (13) over angles of the vector $\mathbf{k}_{\|}$this expression turns to 0 . It does not necessary happen for other than [001] crystal orientations. We will not consider such an opportunity here. Also, we will not discuss here other than Dresselhaus's SOI effects, for example, due to strain, or due to potential gradients along $x, y$ axes. Instead, let us consider a situation when electrons are confined, say, in $y$-direction. In this case $k_{x}^{2}-k_{y}^{2}$ becomes $k_{x}^{2}-\overline{k_{y_{n}}^{2}}$, where the overline and the label $n$ denote averaging of the momentum operator over $n$-th quantum eigenstate in $\mathrm{y}$-direction. Thus, the symmetry between $x$ and $y$ directions is broken and $S_{z}$ does not turn to zero. Below we will assume a parabolic confinement. After averaging over the $y$-direction the spin-orbit interaction (9) depends only on $k_{x}$, with $k_{\|}=\left|k_{x}\right|$.

Substituting (17) into (16) and then into Eq. (15) we express $S_{z}(z)$ through unperturbed eigenstates. The latter are calculated for a square barrier structure described above. For an order of magnitude evaluation of the spin density, the result can be written in an analytical form. Calculations are strongly simplified if only resonance terms in (15) are taken into account. One may also make use of the small parameter $k_{w}^{2} / 2 m_{w} U$, where $k_{w}$ is the wave vector in the $z$-direction within QW and $U$ is the barrier height. At the lowest transmission resonance $k_{w}=k_{0} \approx \pi / 2 d$. By this way, in the leading approximation the spin density in the center of the well $(\mathrm{z}=0)$ and the center of the wire $(\mathrm{y}=0)$ can be written as

$$
\begin{aligned}
S_{z}= & -\frac{4}{\pi} \sum_{k_{x}, n}\left|\phi_{n}(0)\right|^{2} \int_{0}^{\infty} d k \frac{\Gamma^{4}}{\left[\left(k_{w}-k_{0}\right)^{2}+\Gamma^{2}\right]^{2}} \frac{m_{r} m_{b}}{\kappa k} \\
& \times \mathcal{A} \vartheta_{n}(d) h^{2} \cosh ^{2} \kappa b \sinh 2 \kappa b \\
& \times \quad\left[n_{F}^{l}(E)-n_{F}^{r}(E)\right]
\end{aligned}
$$

where $h=k_{x}\left(\beta_{w}-\beta_{b}, \kappa \approx \sqrt{2 m_{b} U}\right.$. The phase $\vartheta_{n}(z)$ is obtained from Eq. (8) by averaging $k_{y}^{2}$ with oscillatory wave functions $\phi_{n}(y)$. The width of the transmission resonance in $k$ space is given by $\Gamma=$ $\left(m_{b}^{2} / m_{r} m_{w}\right)\left(k k_{w} / \kappa^{2} d\right) \sinh ^{-2} \kappa b$, where $m_{r}, m_{b}, m_{w}$ are effective masses in reservoirs, barriers and QW, respectively. $\mathcal{A}$ is a dimensionless function of $k$. The value of this function is close to 1 in the range of parameters under consideration.

For a numerical evaluation the following parameters have been taken: $d=100 \AA, b=40 \AA$, electron density in reservoirs $n=10^{23} \mathrm{~m}^{-3}$, the quantization energy of the parabolic confinement $\hbar \omega=4 \mathrm{meV}, \gamma=27 \mathrm{eV}$ $\AA^{3} \underline{\underline{7}}$ Other parameters correspond to the InAs quantum well, $\mathrm{In}_{0,53} \mathrm{Ga}_{0,47} \mathrm{As}$ barrier, and $\mathrm{In}_{0,9} \mathrm{Ga}_{0,1}$ As reservoirs. With such parameters we calculated $\Gamma=10^{4} \mathrm{~cm}^{-1}$ and $\vartheta(d) \approx 10^{-3}$. From (18), in the linear regime $\Delta \mu=\mu_{l}-\mu_{r} \ll E_{F}$, the spin density can be evaluated as $S_{z} \approx 0,1 \Delta \mu \mathrm{meV}^{-1} \mu \mathrm{m}^{-3}$. Although the spin density is not high, nevertheless, in the range of $1 \mathrm{mV}$ terminal voltages it can be detected by the Kerr rotation method 3 . The parameters of the heterostructure can also be optimized to reach the higher density. Experimentally, the total spin polarization can be enhanced in superlattices.

\section{Spin dependent transmittance}

The second term in (9) causes spin flips of a particle transmitting through the double barrier structure. The spin dependent transmission is obtained from (17) and (16) where $\psi^{l}$ is calculated at $z>d+b$. By this way, near the resonance, the spin flip transmittance becomes

$$
\begin{aligned}
\Delta t_{1,2} & =-i 16 t^{2} \vartheta(d) \mathcal{B} \frac{m_{b}^{3} m_{r}}{m_{w}^{2}} \frac{h^{2} k_{w}^{2}}{\kappa^{3} k} \frac{\cosh ^{3} \kappa b}{\sinh \kappa b}, \\
\Delta t_{2,1} & =-\Delta t_{1,2}
\end{aligned}
$$

where $\mathcal{B} \sim 1$ is a dimensionless factor. It is easy to see that, according to (19), $\Delta t_{1,2}$ transforms an unpolarized flux of electrons, say, from the left reservoir into a flux of spin polarized electrons, with the polarization in zdirection. Indeed, according to our choice of the spin basis, the z-polarization is obtained by taking an average of the $\sigma_{y}$ spin operator. Eq. (19) gives rise to two spinors corresponding to two possible initial spin polarizations: $\psi_{1}=\left(t, \Delta t_{1,2}\right)$ and $\psi_{2}=\left(\Delta t_{2,1}, t\right)$. For an unpolarized source, after summing up averages of $\sigma_{y}$ with these spinors, one obtains a finite result, taking into account that near the resonance $t=i \Gamma\left[k_{w}-k_{0}+i \Gamma\right]^{-1} \approx 1$. It should be noted, that besides (19) $\Delta t_{1,2}$ contains one more term which, however, does not result in a resonant polarized transmission. This term has been neglected. The transmission coefficient obtained from (19) is symmetric with respect to $x \rightarrow-x, y \rightarrow-y$ and, hence, remains finite after the angular averaging, in contrast to a spin filtering effect considered in Ref 9,10 . Close to the Fermi energy and with the same numerical parameters used for evaluation of (18) we obtain $\Delta t_{1,2} \approx 3 \cdot 10^{-3}$. In spite of its small value, this coefficient can lead to a noticeable accumulation of the spin polarization in a reservoir with small spin relaxation rate. For example, let us take a reservoir of $1 \mu \mathrm{m}^{3}$ volume. The spin polarization flux carried from the left reservoir through the wire of the length $L$ is given by

$$
I_{s}=\frac{L}{\pi} \sum_{k_{x}, n} \int_{0}^{\infty} d k \frac{k}{m_{r}} \operatorname{Im}\left[t^{*} \Delta t_{1,2}\right]\left[n_{F}^{l}(E)-n_{F}^{r}(E)\right] .
$$

Taking $\mu_{l}-\mu_{r}=1 \mathrm{meV}, L=1 \mu \mathrm{m}$, the typical spin relaxation time in bulk semiconductors $1 \mathrm{~ns}, \frac{12}{,}$ and all the rest of the parameters as in above evaluations, we get the spin density in the reservoir around $0,5 \mu \mathrm{m}^{-3}$, which is within the optical detection range $\underline{\underline{3}}$ 


\section{CONCLUSION}

We considered the spin orbit effects associated with resonant tunneling of electrons through a symmetric double barrier structure. Two contributions to SOI have been taken into account, namely, the Dresselhaus SOI and the spin-orbit interaction induced by gradients of heterostructure material parameters. We found out that the vertical transport of electrons gives rise to the spin current flowing parallel to heterointerfaces, as well as to the spin polarization within QW. These effects are analogous to the spin-Hall effect and the electric spin orientation, intensively studied recently for 2DEG. A distinction with these traditional studies is that instead of electron wave functions confined in QW we employ eigenstates corresponding to resonant electron transmission. Moreover, instead of an electric field parallel to QW, the vertical bias has been considered. In compliance with such a 3D model, we calculated a distribution in z-direction of the spin-Hall current density and spin polarization. This dependence reveals interesting structure, such as "surface" currents flowing along the heterointerfaces. The spin-Hall current density does not turn to 0 with the external bias, thus signalling about existence of the equilibrium spin current density, which was found to be linear with respect to the spin-orbit interaction. At the same time, in a symmetric double well structure the net equilibrium current, obtained by integration of the current density over $z$, turns to 0 . Although the spin-Hall and the equilibrium currents take place in absence of the Dresselhaus SOI, the latter is necessary to obtain the finite out of plane spin density within QW. It was found out that the most important is a part of the Dresselhaus SOI which is proportional to the $z$-component of the electron momentum operator. This SOI also gives rise to spin dependent transmission. Due to interplay of the Dresselhaus SOI and the spin-orbit interaction induced by gradients of heterostructure material parameters, an unpolarized and isotropic in $x, y$ directions beam of electrons becomes polarized in the $z$-direction after tunneling through double barrier QW.

The effects discussed in this paper can be interesting in application to metal surfaces with strong spin-orbit effects associated with surface states. ${ }^{13}$ The STM set up with a magnetic tip could be employed for studying spin related effects. The above model, however, must be modified to take into account spin-orbit interactions typical for a particular metal surface.

This work was supported by RFBR Grant No 060216699, the National Science Council of ROC under Grant Nos. NSC95-2112-M-009- 004, NSC93-2119M-007-002 (NCTS), and the MOE-ATU Grant.
1 H.-A. Engel, E. I. Rashba, B. I. Halperin, Handbook of Magnetism and Advanced Magnetic Materials, Vol. 5, Wiley (arXiv:cond-mat/0603306); J. Schliemann, Int. J. Mod. Phys. B 20, 1015 (2006).

2 J. Wunderlich, B. Kaestner, J. Sinova, and T. Jungwirth, Phys. Rev. Lett. 94, 047204 (2005).

3 Y. K. Kato, R. C. Myers, A. C. Gossard, and D. D. Awschalom, Science 306, 1910 (2004)

4 V.M. Edelstein, Solid State Commun., 73, 233 (1990); F. T. Vasko, N. A. Prima, Sov. Phys. Solid State, 21, 994 (1979); E. L. Ivchenko, G. Pikus, JETP Lett. 27, 604 (1978); L. S. Levitov, Y. N. Nazarov, G. M. Eliashberg, Sov. Phys. JETP, 61, 133 (1985); J. I. Inoue, G. E. W. Bauer, and L.W. Molenkamp, Phys. Rev. B 67, 033104 (2003)

${ }^{5}$ G. Dresselhaus, Phys. Rev. 100, 580 (1955)

6 E. A. de Andrada e Silva, G. C. La Rocca, F. Bassani, Phys. Rev. B 55, 16293 (1997)

7 R. Winkler, Spin-Orbit Couplig Effects in Two- Dimen- sional Electron and Hole Systems (Springer, Berlin, 2003).

8 Yu. A. Bychkov and E. I. Rashba, J. Phys. C 17, 6039 (1984)

9 A. Voskoboynikov, S. S. Liu, and C. P. Lee, Phys. Rev. B 59, 12514 (1999); E.A. de Andrada e Silva and G.C. La Rocca, Phys. Rev. B 59, 15583 (1999).

10 V.I. Perel', S.A. Tarasenko, I.N. Yassievich, S. D. Ganichev, V. V. Bel'kov, and W. Prettl, Phys. Rev. B 67, 201304 (2003); M. M. Glazov, P. S. Alekseev, M. A. Odnoblyudov, V. M. Chistyakov, S. A. Tarasenko, and I.N. Yassievich, Phys. Rev. B 71, 155313 (2005); L. G. Wang, Wen Yang, and Kai Chang, Phys. Rev. B 72, 153314 (2005).

11 E. I. Rashba, Phys. Rev. B 68, 241315 (2003)

12 I. Malajovich, J. M. Kikkawa, and D. D. Awschalom, Phys. Rev. B 84, 1015 (2000)

13 C. R. Ast, et. al. Phys. Rev. Lett. 98, 186807 (2007) 